\title{
La educación física como fortalecimiento de valores ciudadanos para la convivencia Physical education as a strengthening of citizen values for coexistence
}

\author{
Felix Giovanni Cañon Salinas, Mario Alberto Villarreal Ángeles \\ Universidad Montrer (México), Universidad Juárez del Estado de Durango (México)
}

\begin{abstract}
Resumen. Esta investigación surge de la problemática que se enfrentan ante la pérdida de valores entre los jóvenes y la necesidad de mejorar su comportamiento, empleado el área de Educación Física como estrategia para el fortalecimiento de los valores ciudadanos y la convivencia en instituciones educativas oficiales de Ibagué, Colombia. El propósito del estudio fue conocer el nivel de actitud y percepción que tienen los estudiantes de básica secundaria con relación a la práctica de valores al momento de la clase y poder precisar la contribución de la Educación Física en su consolidación y fortaleza y, así construir, un plan de acción para que el ejercicio de valores ciudadanos sea cotidiano, tanto en la clase como en el entorno escolar y en su vida social. Empleado una metodología de carácter cualitativo - cuantitativo con enfoque ciberetnográfico, a través de un plan de auto-observación, se pudo detectar comportamientos en formación de valores de los estudiantes de sexto a noveno grado, frente a los patrones de formación ciudadana expresados al recibir clases de educación física. Los resultados indican una actitud positiva hacia la asignatura, percibiendo la práctica de los valores de honestidad, respeto, transparencia, responsabilidad, tolerancia, justicia y participación ciudadana, entre otros, como las principales convicciones que vienen determinado su forma de ser y orientan su comportamiento social; situación que se vigoriza con las actividades desarrolladas en clase y consolidadas con un plan de acción liderado por todos los docentes para la utilización de alternativas pedagógicas que sustenten una formación ciudadana integral.
\end{abstract}

Palabras Clave: Actitud, Educación Física, Valores ciudadanos, Convivencia, Participación ciudadana, Comportamiento social.

Summary. This research arises from the problem faced by the loss of values among young people and the need to improve their behavior, using the area of physical education as a strategy to strengthen citizen values and coexistence in official educational institutions of Ibagué, Colombia. The purpose of the study was to know the level of attitude and perception that elementary school students have in relation to the practice of values at the time of class and to be able to specify the contribution of physical education in its consolidation and strength and, thus, build, an action plan so that the exercise of civic values is daily, both in the classroom and in the school environment and in their social life. Employed a qualitative-quantitative methodology with a cybernetic approach, Through a self-observation plan, it was possible to detect behaviors in the formation of values of students from sixth to ninth grade, compared to the patterns of citizenship formation expressed when receiving physical education classes. The results indicate a positive attitude towards the subject, perceiving the practice of the values of honesty, respect, transparency, responsibility, tolerance, justice and citizen participation, among others, as the main convictions that determine their way of being and guide their behavior. Social; a situation that is invigorated with the activities developed in class and consolidated with an action plan led by all teachers for the use of pedagogical alternatives that support a comprehensive civic education.

Keywords: Attitude, Physical Education, Citizen values, Coexistence, Citizen participation, Social behavior.

\section{Introducción}

El desarrollo de las competencias sociales y ciudadanas en los escolares está relacionado con una de las razones de la educación como es la formación de ciudadanos idóneos en el ejercicio una ciudadanía justa y activa, capaces de establecer relaciones y de mostrar una actitud responsable y creativa en el momento que tengan que enfrentar conflictos naturales de convivencia

Fecha recepción: 04-08-20. Fecha de aceptación: 04-11-21

Felix Giovanni Cañon Salinas

fcanon@unimontrer.edu.mx
(Comte, 2013, citado en Monzonis, 2015, p.18). Es así como, el área de educación física (EF), al alcanzar en los últimos años una gran solidez curricular permite estar a la altura de otras materias. Desde el punto de vista recreacional, se convierte en el escenario ideal para generar y consolidar hábitos de práctica saludables que les permitan a los estudiantes actuar de manera autónoma en competencias básicas y capacidades atléticas, motrices, sociales y ciudadanas con el fin de interaccionar con su entorno y sobre todo para adquirir los valores necesarios para la vida en sociedad.

Ahora bien, la fortaleza educativa de la EF y el deporte con relación al desarrollo de los valores sociales y 
ciudadanos está inmersa en los marcos normativos y curriculares que la orientan y en el contexto cultural de las comunidades. Resulta contradictorio la cantidad de comentarios que se hacen sobre el desarrollo y manifestaciones de valores sociales a través de la EF, respecto a las referencias científicas que se encuentran en la literatura especializada (Gutiérrez, 1995). Por esta razón, para intentar comprender la EF orientada a los valores, se debe situarse en la naturaleza misma del ser humano, identificando su desarrollo físico, actitudes y habilidades culturales primarias. A lo largo de la historia, la actividad física ha desempeñado un papel fundamental en la cotidianidad humana. Sin embargo, hay que partir de hechos recientes que avalan la importancia de la EF para el progreso humano en general, de los niños y jóvenes.

En el artículo sobre «el valor como contenido de la educación física escolar», Miraflores (2006), citando a Ortega y Mínguez (2001), presentó una propuesta práctica de aplicación en la escuela para la formación en valores, fundamentada en cinco modelos teóricos concretos, basados en ideas de diferentes de ideólogos, pensadores y pedagogos de reconocida trascendencia, así:

1) La Educación moral como formación del carácter (Lickona, 1991), donde los niños aprenden principios morales y valores haciendo practica la teoría, es decir, que forme parte de ellos y que exploren gracias a la práctica en su vida cotidiana con familiares y amigos.

2) La educación moral como clarificación de valores (Raths, 1966). Se les capacita para que decidan y disciernan lo bueno y lo malo, bajo un proceso reflexivo y autónomo.

3) La educación moral como desarrollo del juicio moral (Dewey, Piaget y Kohlberg, 1975). Los niños toman conciencia de los problemas éticos sociales, gracias a los conflictos generados, el diálogo con los demás, la búsqueda del respeto y la tolerancia, la estimulación a través de la actividad física como práctica aplicada.

4) Búsqueda del desarrollo de la autoestima (Purkey y Novak, 1984; Harter, 1986). Se obtiene un refuerzo de su autoestima a partir de la aplicación práctica de determinadas pautas, normas, reglas y actividades. Todas ellas coordinadas por el docente y apoyadas por los padres.

5) Reforzamiento del alumno en el contexto de la responsabilidad social (Hellison, 1973). Adquieren un sentido de responsabilidad social gracias a que el profesor regula las situaciones mediante grupos reducidos, con metodologías basadas en la resolución de problemas, conversaciones, propuesta de dilemas, toma de decisiones, etc. (p. 150)

En referencias recientes, la tesis doctoral de Velásquez (2013), plantea que, en el deporte se presentan oportunidades para conocer el comportamiento ético de los estudiantes, pues permite encontrar si la conducta mostrada es correcta o incorrecta. Es allí, donde se aprecia que la EF es una oportunidad real y válida para promover valores sociales. Es un área de alto potencial educativo para que los alumnos adquieran valores y desarrollen actitudes que socialmente hoy en día son necesarias, por lo cual la enseñanza debe tener ese fin único, siendo necesario que el profesor esté comprometido y busque momentos de diálogo para el análisis y crítica sobre situaciones, actitudes y conductas que se suceden en la actividad física y el deporte. Así mismo, el estudio sobre «la educación física como entorno de trabajo de la educación en valores en educación primaria» desarrollado por Igarabide (2014), plantea un análisis teórico sobre el potencial que tiene el área de EF para promover valores y el compromiso del docente para alcanzar estos propósitos, aprovechando los constantes dilemas que surgen en el deporte o en la actividad física para que los estudiantes opten por un proceder acorde con los valores compartidos socialmente. El acatamiento de las normas de juego y los rivales hace que prevalezcan los valores como la igualdad, la tolerancia, la amistad, la solidaridad, el juego limpio y el respeto que harán en el futuro un ciudadano de bien.

Por eso, en la EF están dadas las mejores condiciones para introducir en sus actividades la formación ciudadana, los procesos de organización, la participación, la práctica de valores como la solidaridad, la autonomía, la responsabilidad y el respeto a través de los juegos, las danzas, los deportes y la gimnasia, entre otros. El Ministerio de Educación Nacional (MEN) (1996), «los comportamientos que dan cuenta de los valores, y brindan las condiciones que hacen posible la reflexión y el diálogo para reafirmar algunos comportamientos o modificar otros a través de acuerdos sobre reglas de Juego» (p.39).

Son múltiples los factores que son determinantes al momento de acometer la tarea educativa en la clase de EF desde el ámbito ético. Aparecen diferentes elementos que ayudan a configurar el perfil propio de esta área curricular, permitiendo encausar de forma explícita, constructiva e intencionalmente la educación en valores, los cuales se pueden identificar en la figura 1, tal como lo propone Ruiz (2014).

Esto permite considerar ocho aspectos relevantes: En primer lugar, el cuerpo y el movimiento como ejes de la acción pedagógica, lo cual desvirtúa la idea de la 




EF centrada solamente en los aspectos físicos y corporales, sino que también educa en, desde y a través de la motricidad, condicionado así, los valores de carácter personal, ético y estético que acercan a la singularidad individual. Un segundo hecho es que, la EF está estrechamente ligada al especio vivencial de niños y adolescentes, dándole significado a su vida y proporcionándole sentido a sus experiencias al situarlos en los dilemas que atañen a la esfera de los valores. El tercer elemento es la convergencia de intereses de lo vivido por los estudiantes fuera de la institución educativa, lo que hace especial este trabajo investigativo al tener en cuenta que la convergencia axiológica entre las actividades practicadas en diferentes contextos. Un cuarto campo es el inherente al carácter explícito de las conductas motrices, lo cual influye en forma directa sobre el comportamiento ético de los estudiantes, por la importancia que tiene en el desarrollo de la autoestima, elemento íntimamente ligado a la pedagogía de los valores. El quinto factor tiene que ver con la implicación afectiva de las actividades motrices, convirtiéndolas en un medio decisivo para el desarrollo socio afectivo y la educación ética, lo cual permite aprender a desenvolverse en el mundo actual, planteándose retos y lograr superar dificultades y frustraciones (Ruiz, 2014).

En este mismo orden de ideas, el sexto elemento influyente en la formación de valores en la EF tiene que ver con el componente racional, el cual marca las actividades motrices que se desarrollan en las clases en donde se resalta la comunicación y el intercambio, forjadoras de las actitudes de colaboración, reciprocidad en la ayuda, atención a las necesidades, equidad, enfrentamiento, superioridad, autoritarismo o subordinación, las cuales atañen al ámbito de los valores. De igual manera, el área de EF tiene un marcado carácter social en donde prevalece los valores de libertad, responsabilidad, respeto, cooperación o solidaridad que ayudan y fortalecen el crecimiento personal del estudiante y su convivencia humana, como también valores de eficacia, competitividad, éxito o superioridad, ambivalencia que permite enfocar críticamente su influencia (Ruiz, 2014).

$\mathrm{Al}$ observar una clase de EF, son múltiples los detalles que hacen pensar en distintos valores, diferenciando los que le corresponden al docente en cuanto a los objetivos y la metodología empleada; los que conciernen a los estudiantes en sus actitudes y el desempeño de su tarea, todo esto hace parte de la política educativa. De esta manera se puede apreciar que, los contenidos de la clase pretenden buscar el rendimiento físico, objetivos relacionados con la salud, y los valores de tolerancia, lo que se debe preguntar cuál es el tratamiento de género, la importancia de la teoría y la práctica con las demás áreas del currículo.

El dinamismo característico de la clase de EF y el constante desplazamiento de los escolares en el área deportiva, exigen la utilización de procedimientos organizativos tradicionales y modernos, que permitan reorganizarlos en la clase. Una adecuada preparación del material incide favorablemente, en el aprovechamiento del tiempo y el rendimiento de la misma, estos índices permiten evaluar la eficiencia del proceso de enseñanza-aprendizaje, en función de contribuir a la formación ciudadana. (Escalante, 2014, p. 2)

Para el logro de estas metas, este mismo autor se vale de ciertas fuentes y medios que, dependiendo de su enfoque, ha variado su concepción y énfasis con el tiempo. Sin embargo, lo que es incuestionable, son las aportaciones que la práctica de la EF ofrece a la sociedad contribuyendo al cuidado y preservación de la salud, al fomento de la tolerancia y el respeto de los derechos humanos, la ocupación del tiempo libre y el impulso de una vida activa en contra del sedentarismo. Los medios utilizados para tal propósito son el juego motor, la iniciación deportiva, el deporte educativo y la recreación. (Escalante, 2014, p.3)

Con esto, se logrará desarrollar competencias que sean el resultado de la integración de métodos y medios potenciadores de capacidades motrices y ciudadanas que permitan un desempeño ciudadano exitoso.

\section{Metodología}

Atendiendo a los objetivos y metas investigativas, 
esta investigación se enmarcó en una orientación cualitativa, con diseño etnográfico, como lo aconseja Goetz y LeCompte (1988), permitió observar y recolectar evidencias directamente en la realidad de la situación abordada y con fuentes primarias, para llegar a analizar e interpretar los resultados de estas indagaciones. Además, el trabajo se apoyó en el paradigma hermenéutico, convirtiendo la observación en un ejercicio cotidiano para ir registrando las diferentes acciones y acercarse a la interacción contextual. Esta construcción dialógica de la realidad se tenía previsto mediante la observación participante, a través del registro, en el espacio/tiempo de la clase de EF como formadora de valores ciudadanos para convivencia desde la vida misma de los estudiantes de secundaria y los docentes.

Aunque en la investigación cualitativa la interacción personal para la recolección de datos es muy relevante, el uso masivo de Internet en todas las actividades diarias ha llevado a comprender que se está en la era de la e-investigación y como lo señala (Hine, 2004; Sánchez \& Ortiz, 2017), fue el momento de orientar la etnografía hacia la netnografía o ciberetnografía, con una visión rigurosamente crítica, permitiendo el uso de los recursos de la Red en los procedimientos de investigación educativa. Así, esta investigación cualitativa-cuantitativa, optó por la etnografía virtual y las herramientas de Internet o redes sociales para realizar procesos de auto- observación, video-entrevistas, grupos focales en red; técnicas que hacen posible el trabajo investigativo sin necesitar de la relación presencial, usando medios como e.g. (cámaras del ordenador, Smartphone, cámaras de video o alternativas Skype, Facebook, WhatsApp, Instagram y Google forms).

La población objeto de la investigación estuvo integrada por 655 estudiantes de los grados sexto, séptimo, octavo y noveno de la institución educativa técnica oficial de la ciudad de Ibagué, en el departamento del Tolima, Colombia. El tipo de muestra fue por conglomerados, esto es por aula de clase o grupo-clase, la cual se seleccionó de manera intencionada, eligiendo un grupo por cada uno de los grados de básica secundaria y sus respectivos directores de grado, para un total de $\mathrm{n}=144$ estudiantes y $\mathrm{n}=5$ docentes, incluyendo al coordinador académico del colegio.

Un primer instrumento empleado fue un cuestionario tipo encuesta virtual para valorar la actitud de los estudiantes en varios momentos de la clase de EF con indicadores de frecuencia tipo Likert. Constó de 24 preguntas agrupadas en cuatro aspectos: el primero relacionado con el comportamiento para conocer el nivel de convivencia, comportamiento, responsabilidad, honestidad e interés frente a la clase; el segundo referente a las normas y reglamentos detectando el grado de aceptación, moral, justicia y cumplimiento de reglas de juego; el tercero sobre las ayudas y cooperación entre compañeros, indagando grado de colaboración, valoración, respeto, tolerancia, solidaridad y participación ciudadana con los compañeros de clase; finalmente, un dominio relacionado a la clase de EF frente a su aceptación, perseverancia y transparencia. El nivel de actitud obtenido se categoriza como alto, medio o bajo para el análisis de resultados.

El segundo formulario fue una auto-observación de estudiantes, elaborado ad hoc, con 36 ítems, a través de la escala de frecuencia entre siempre a nunca determino el nivel de convivencia, responsabilidad, honestidad, tolerancia, respeto, cooperación, solidaridad, justicia, participación ciudadana, transparencia, como valores ciudadanos. Esta ficha constó de tres partes: la primera identifico al estudiante auto-observado, la segunda se conformó por tres columnas en los que se establece los valores ciudadanos, los indicadores con cuatro niveles de frecuencia y, la última parte, una planilla de registro de la puntuación obtenida para determinar el total acumulado por respectivo curso del grado observado. Un tercer instrumento empleado para complementar la información de estudiantes fue una entrevista virtual a docentes orientadores del área acerca de la práctica de los valores ciudadanos, con once preguntas abiertas frente al significado del área de EF en lo relacionado con la formación en valores, la importancia de esta temática y la forma como está influyendo en la educación de sus estudiantes. Luego, se desarrolló un grupo de discusión virtual, donde participaron nueve (9) personas, cuatro estudiantes y cinco profesores del área y el investigador como moderador.

Los instrumentos diseñados para la investigación en particular, se sometieron al proceso de validación de su contenido por juicio de expertos, teniendo en cuenta su experiencia, trayectoria investigativa y la familiaridad con la temática de formación en valores ciudadanos. Luego de recibir las sugerencias y observaciones se elaboraron los cuestionarios definitivos para ser suministrados a los estudiantes y docentes.

El análisis cuantitativo se hizo para el cuestionario a estudiantes y el formato de observación no participante, estableciendo un código para cada elemento y proceder así al tratamiento con el software de análisis estadístico Microsoft Excel y XLSTAT, por lo que ambas matrices de datos fueron configuradas con el editor de 
datos del programa. Una vez elaboradas estas matrices se siguió el procedimiento estadístico descriptivo, expresando los datos reunidos en forma representaciones gráficas de barras o de sectores, tanto para variables categóricas o de contingencia. De todas maneras, se optó por un análisis estadístico exploratorio-descriptivo obteniendo la distribución de frecuencias y de variabilidad de datos eficaz y práctico, entregando información clara y entendible acerca de la forma como la clase de EF favorece el fortalecimiento de los valores ciudadanos en los estudiantes de secundaria.

Para que la información fuera comprensible y operativa y respondiera a cada una de las preguntas de investigación se dispuso de un cuadro matriz para establecer relaciones entre las categorías y los significados de los resultados obtenidos para asegurar que la información reunida fuera eficaz y mostrara el nivel de logro de los objetivos planteados y poder así, establecer unas conclusiones respaldadas sólidamente.

\section{Resultados}

\section{Actitud hacia los valores en la clase de EF.}

El primer objetivo planteado fue describir la actitud de estudiantes con relación a los valores ciudadanos en clase de EF. Naturalmente, fue necesario elegir una serie de momentos en los se conjugan aquellas situaciones en las que se podía conocer la predisposición de los alumnos para comprobar sus reacciones y comportamientos, favorables o no, frente a la realidad vivida en cada una de sus clases, particularmente en los aspectos de convivencia, comportamiento personal, responsabilidad, honestidad y el interés que muestra hacia la clase.

En cuanto a la actitud comportamental hacia la clase de $E F$, los estudiantes de básica secundaria muestran una mayor actitud positiva cuando manifiesta confianza al realizar los ejercicios o prácticas deportivas con el $72 \%$,

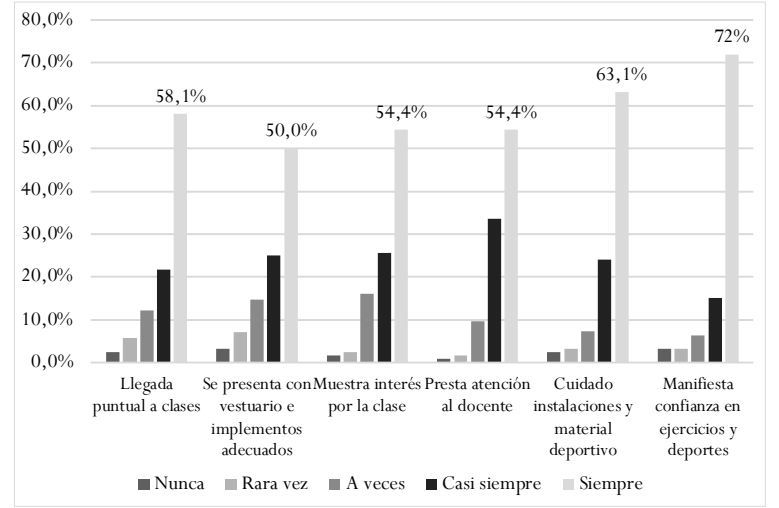

Figura 2. Actitud comportamental de los estudiantes en la clase de Educación Física. Fuente: (IESF, Ibagué, 2020) seguido del cuidado a las instalaciones y el material deportivo $(63,1 \%)$, la llegada puntual a clase con el $58,1 \%$; luego, el prestar atención a las indicaciones de sus profesores e instructores y mostrar interés por aprender, cada uno con el $54,4 \%$ y por último la presentación con el vestuario y los implementos deportivos adecuados, con el $50 \%$.

Frente a la actitud hacia normas y reglamentos, se comprobó cómo, la mayoría de los estudiantes expresan actitud positiva hacia las normas y reglas de juego vigentes en la clase de EF y en la práctica deportiva, tal como se comprueba a través de la figura 3, cuando el 75,7\% de los alumnos encuestados señala que siempre acepta y respeta las normas y reglas de juego, el 71,5\% siempre acata las decisiones e indicaciones de sus profesores y un $68,1 \%$ advierte que el ganar o cumplir los objetivos de la clase, únicamente tienen mérito y valía cuando hay respeto por las reglas y normas establecidas. No obstante, un menor nivel de actitud se observó frente a los juicios sin favorecimiento que emiten los profesores y árbitros $(47,9 \%)$ y el no tratar de engañar ni romper reglas en beneficio propio $(43,1 \%)$

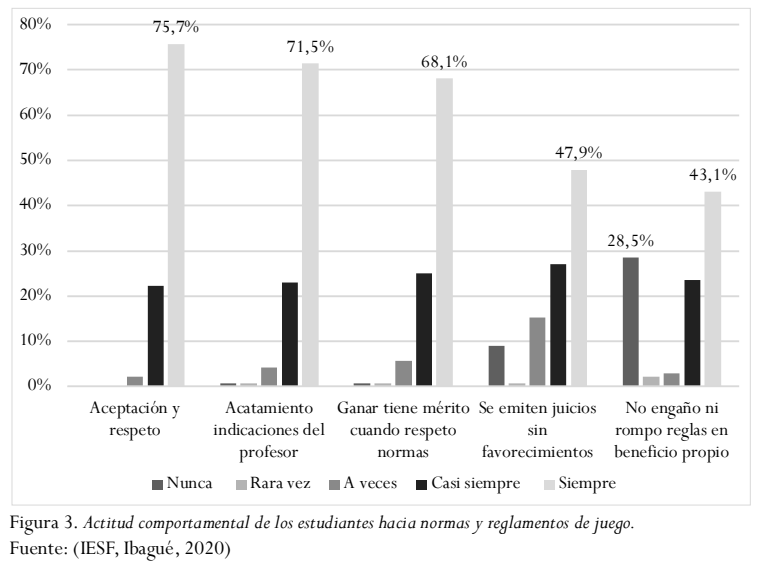

En ayuda y cooperación entre compañeros, la actitud de los estudiantes es positiva, según lo reflejan los siguientes datos: el 69,4\% siempre valora los logros de sus compañeros, el 61,8\% muestra a todo momento su compañerismo, el 56,9\% acepta con agrado y sin disgustarse otros puntos de vista, un 54,9\% ayuda a sus compañeros en dificultad, el $50 \%$ se declara tolerante y, en menor escala, el 42,4\% dice aceptar comportamientos ajenos sin fastidiarse, en tanto el $41,7 \%$ asegura que siempre está dispuesto a colaborarle a su profesor para el desarrollo de la clase (figura 4). Acá, cabe resaltar que todos los estudiantes valoran los logros de los demás y en la práctica deportiva o física, siempre muestran valores de compañerismo. 




Con relación a la actitud hacia la clase de EF se conoció el grado de aceptación, perseverancia y trasparencia que los estudiantes de secundaria tienen por la clase. Así, el 59\% aseguró que siempre se permite la participación en actividades físicas, un $65,3 \%$ adquieren valores sociales y ciudadanos para una mejor convivencia escolar y grupal. Además, tal como se refleja en la figura 5, el 64,6\% cree que esta clase siempre es importante para su desarrollo personal, un 63,9\% opina que esto se debe al ánimo que reciben de sus profesores a participar en las actividades físicas y deportivas. Sin embargo, preocupa que el 30,6\% aseguren que los docentes nunca les hacen caso a sus insinuaciones y sólo el 16,7\% dice contar siempre con atención de sus docentes.

\section{Percepción de valores ciudadanos en la clase de $\mathbf{E F}$}

Teniendo como punto de partida el cuestionario de auto-observación diligenciado por estudiantes acerca del nivel de percepción sobre cumplimiento de valores de convivencia, responsabilidad, honestidad, tolerancia, respeto, cooperación,

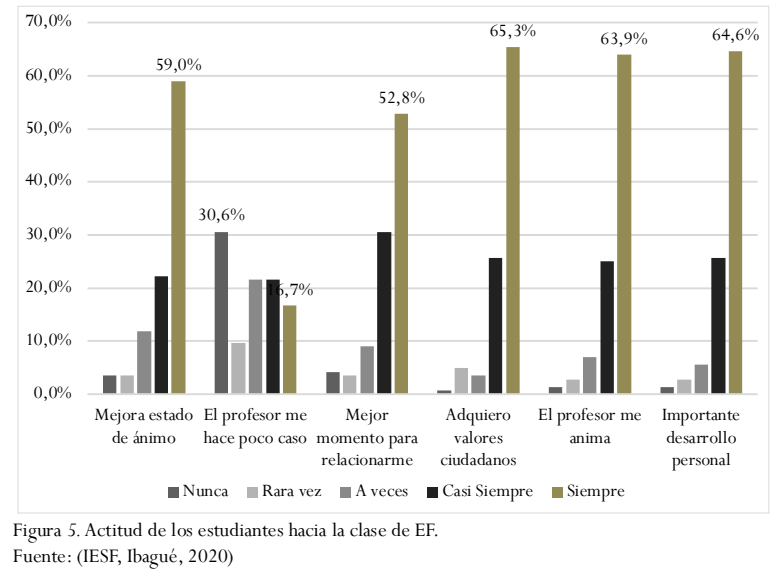

solidaridad, justicia, participación ciudadana, transparencia y probidad. Los resultados que muestra la Tabla 1 permitieron establecer que, frente al valor de convivencia, el 83,3\% de los estudiantes tienen un nivel positivo de armonía, lo cual asegura una relación auténtica entre los compañeros y le da a la EF la posibilidad de ser un área que trasciende hacia el fortalecimiento de sus valores sociales y ciudadanos, preparándolos para enfrentar con optimismo su futuro. Además, en la puesta en práctica de la responsabilidad, la mayor parte de los estudiantes $(83,4 \%)$ dan muestras de ser responsables porque hacen uso adecuado de los implementos deportivos, cumplen con las actividades programadas y muesTabla 1.

\begin{tabular}{|c|c|c|c|c|}
\hline \multirow[t]{2}{*}{ Valores } & \multirow[t]{2}{*}{ Indicadores } & \multicolumn{3}{|c|}{$\begin{array}{c}\text { Porcentajes } \\
\text { Nivel de Percepción }\end{array}$} \\
\hline & & Bajo & Medio & Alto \\
\hline Convivencia & $\begin{array}{l}\text { Respeta horarios y reglas de juego. } \\
\text { Respeta turnos correspondientes } \\
\text { Se relaciona con compañeros sin preferencias. } \\
\text { Muestra autonomía en actuaciones. }\end{array}$ & 6,6 & 10,1 & 83,3 \\
\hline Responsabilidad & $\begin{array}{l}\text { Muestra una actitud positiva hacia sus compañeros y rivales. } \\
\text { Responde correctamente por todas las acciones rutinarias. } \\
\text { Cuida y hace buen uso del material } \\
\text { Cumple con sus obligaciones escolares y físicas. }\end{array}$ & 5,9 & 10,7 & 83,4 \\
\hline Honestidad & $\begin{array}{l}\text { Evita romper normas para beneficio propio. } \\
\text { Asume la autoría de faltas y conductas negativas. } \\
\text { Dice la verdad } \\
\text { Es honrado y no hace trampa }\end{array}$ & 5,5 & 10,1 & 84,4 \\
\hline Tolerancia & $\begin{array}{l}\text { Valora y acepta al grupo al que pertenece. } \\
\text { Apoya y anima a sus compañeros cuando se equivocan. } \\
\text { Acepta con agrado a sus compañeros en grupos de trabajo o de juego. } \\
\text { Fomenta el buen ambiente escolar, aceptando errores en compañeros. }\end{array}$ & 5.7 & 10,1 & 84,2 \\
\hline Respeto & $\begin{array}{l}\text { Ayuda a sus compañeros cuando tienen dificultades con sus tareas. } \\
\text { Reconoce los triunfos de los demás. } \\
\text { Respeta a los adversarios cuando ganan o pierden. } \\
\text { Entiende la competición como forma de hacer amigos. }\end{array}$ & 5,6 & 9,9 & 84,5 \\
\hline Cooperación & $\begin{array}{l}\text { Respeta a sus compañeros y docentes. } \\
\text { Aporta esfuerzo y destreza para ayudar al grupo o a un compañero. } \\
\text { Cuida, colabora y hace buen uso del material de trabajo. } \\
\text { Ayuda a mantener la armonía del grupo. }\end{array}$ & 5,9 & 10,6 & 83,5 \\
\hline Solidaridad & $\begin{array}{l}\text { Apoya a los compañeros cuando se encuentran en dificultades. } \\
\text { Auxilia a los compañeros cuando se lesionan o sufren un accidente. } \\
\text { Anima a sus compañeros cuando se equivocan. } \\
\text { Valora las capacidades de los demás. }\end{array}$ & 6,4 & 10,8 & 85,8 \\
\hline $\begin{array}{l}\text { Justicia / } \\
\text { Participación } \\
\text { Ciudadana }\end{array}$ & $\begin{array}{l}\text { Antepone el bienestar general al particular. } \\
\text { Actúa con justicia e imparcialidad. } \\
\text { Es equitativo y justo en sus actuaciones. } \\
\text { Valora los resultados como consecuencia del trabajo en grupo. }\end{array}$ & 6,4 & 11,0 & 82,6 \\
\hline $\begin{array}{l}\text { Transparencia/ } \\
\text { Probidad }\end{array}$ & $\begin{array}{l}\text { Entrega información veraz y no miente para sacar provecho. } \\
\text { Respeta los derechos de los demás. } \\
\text { Reconoce sus errores y no hace trampa para ganar. } \\
\text { Practica el juego limpio. }\end{array}$ & 6,5 & 10,7 & 82,8 \\
\hline
\end{tabular}

tran actitudes positivas hacia sus compañeros; sin embargo, existen casos en los que se debe intervenir para implementar acciones para el fortalecimiento del modo de actuar responsable a través de la clase, seguramente porque no conocen su importancia, y no estar motivados o no entender sus alcances y potencialidades.

Con referencia a la percepción de honestidad se descubre estudiantes honrados, no hacen trampa durante las actividades, tareas o deportes, evitan violar normas y reglamentos de juego en beneficio propio, asumen la autoría de faltas o conductas negativas y generalmente dicen la verdad. Los estudios sobre la autoevaluación de los estudiantes respecto a su propia conducta revelaron 
que, aunque es difícil evaluar concretamente los valores éticos de una persona, la EF influye positivamente en la formación moral y social de los alumnos, particularmente cuando se le pide auto-observar la frecuencia de sus actos de honestidad. En cuanto a la tolerancia, los estudiantes muestran respeto por las opiniones diferentes, no vulneran los derechos humanos y entienden que todos son diferentes, nadie es más que otra persona, ni está en condiciones de juzgar a otros ciudadanos. En el valor del respeto se ve que, en el escenario educativo se tienen en cuenta los sentimientos y necesidades de los demás, se respeta la opinión de compañeros en diferentes contextos, escuchan y saben esperar, controlan la impaciencia cuando no consigue lo deseable, piden perdón si no trata bien a otros y ayuda a compañeros, reclamando que se les respete, aunque, con alguna frecuencia, les cuesta trabajo hacer amigos a través del juego, seguramente porque se disgustan si no consiguen sus propósitos. El estudiantado colabora en las iniciativas de solidaridad y ayuda a los demás, aunque lo hace, no por hacerse notar sino por convicción. Sin embargo, a veces se debe animarlos a asumir responsabilidades, aunque, habitualmente se comportan de manera correcta con sus compañeros de grupo, dan muestras de sensibilidad ante sus necesidades y problemas o llevando a la práctica sentimientos de solidaridad y ayuda mutua, a pesar que, en algunos momentos de la clase, se deba incentivarlos y animarlos a ser mejor compañeros, más sensibles y a compartir como amigos para el logro de una sana convivencia y el bien común.

El dinamismo característico de la clase de EF y el constante desplazamiento de los estudiantes en el área deportiva permiten desarrollar competencias y comportamientos que sean resultados de la integración de métodos y medios potenciadores de competencias motrices y ciudadanas que permitan un desempeño ciudadano exitoso. La eficiencia del proceso de enseñanzaaprendizaje y la preparación adecuada del material contribuyen al fomento del respeto de los derechos humanos, el impulso de una vida activa, a cumplir los reglamentos de buena fe, a seguir las reglas, a evitar acusaciones negligentes y no abusar de los demás, a diferenciar entre lo que es justo y lo que no, a tener disposición de ayudar, a respetar las cosas y colaborar gustosamente por el bien común, a salir en defensa de otros si considera que han sido víctimas de una injusticia y, en la medida de sus posibilidades, a trabajar por conseguir lo que cree justo para él, para el grupo o la sociedad. Alguien decía que no se requieren leyes y reglamentos ni tampoco policías o fiscales para que la persona actúe en forma transparente y honesta; pero si, una sólida formación valórica y ciudadana que ayude a enfrentar los dilemas éticos que le surgen a diario. La EF cumple un rol fundamental en la formación de valores ciudadanos, cuando los estudiantes reconocen sus derechos y los principios que deben orientar sus actos y los de terceros, en especial la transparencia y la probidad.

\section{Entrevista virtual}

Con relación a las actitudes de los estudiantes durante las clases los docentes entrevistados entregan una valoración positiva ya que reconocen una buena disponibilidad de participación, atención, respeto, integración, alegría, de convivencia pacífica, de pasar momentos agradables y de compartir con sus compañeros. Además, se logró identificar, gracias a los docentes, que en las clases de EF de la institución educativa sobresalen los valores de tolerancia, juego limpio, honestidad, compañerismo, solidaridad, puntualidad, responsabilidad, respeto, igualdad, fraternidad, compromiso y resiliencia.

\section{Grupo de discusión}

El papel de la clase de EF en la formación de valores ciudadanos no se puede orientar solamente hacia el estudiante, sino que también tiene que ser formado el docente del área, el director de grupo, el padre de familia, para que entre todos valoren este espacio de formación junto a las demás áreas y a la misma institución educativa de manera integral. Y es que la clase de EF percibida, es sentida desde los valores ciudadanos que orienta al ser y hacer en la cotidianidad de cada uno de los estudiantes de secundaria.

\section{Plan de acción para el ejercicio de los valores en la clase de EF}

El plan de acción para fortalecer los valores ciudadanos a través de la clase de EF, está fundamentado en la propuesta de Miraflorez (2006), esta propuesta fue adaptada a las características propias de los estudiantes de secundaria y tuvo como objeto poner de manifiesto la importancia de los valores en el ámbito escolar y por consiguiente en la clase de EF, logrando hacer partícipes a todos los estamentos educativos, utilizando el concepto de valor como contenido y mostrar una forma, novedosa y aplicada, de adquirir valores sociales y ciudadanos a través de la clase de EF.

El profesor o el instructor quien usando una guía metodológica permitió desarrollar el plan de acción, teniendo presente que las edades de aplicación serán de 10 a 15 años. Los contenidos de valores a desarrollar y 
potenciar, fueron los siguientes: amistad, generosidad, sinceridad, justicia, compañerismo, honestidad, esfuerzo, deportividad, respeto, solidaridad, responsabilidad, compromiso, orden, convivencia, autoestima, tolerancia, participación ciudadana y probidad.

Por otra parte, la narración de anécdotas propicio la reflexión colectiva, la discusión de dilemas morales, el análisis de los problemas éticos que amenazan el deporte, la orientación de actividades extraescolares vinculadas a los valores y el deporte, la realización de competencias entre diferentes grupos del mismo grado, las lecturas de periódicos y revistas sobre artículos de actitudes asumidas por deportistas y la proyección de películas que reflejen la expresión de valores ciudadanos y su importancia para la vida.

\section{Conclusión y discusión}

La actitud de los estudiantes de secundaria ante la clase de EF se clasificó en positiva, neutra o negativa. En cuanto a la actitud frente al comportamiento en clase, se logra establecer una actitud favorable por parte de los estudiantes hacia la clase de EF, donde, aproximadamente 7 de cada 10 alumnos muestran una actitud positiva porque, llegan puntual a clase, se presentan con la indumentaria apropiada para el desarrollo de las actividades físicas y deportivas, muestran interés por aprender, escuchan con atención las instrucciones de sus docentes e instructores, pero, sobre todo, son muy cuidadosos con el material deportivo y se sienten seguros y confiados al realizar las actividades.

La importancia de la clase de EF no está solo vinculada a una actitud de relajación y desinhibición y de relax, sino que, además de ser un acto pedagógico, viene dada como un escenario de convivencia social donde afloran conductas que permiten evidenciar la puesta en práctica que tienen los estudiantes con relación a los valores. Su importancia no es afín solo a un tiempo de descanso de las actividades curriculares diarias o, como lo afirma un docente, «un espacio para controlar y mantener la salud mental y física del ser humano, de hacer actividad deportiva», su importancia viene dada como espacio de convivencia social, donde surgen comportamientos que permiten evidenciar la puesta en práctica que tienen los estudiantes con relación a los valores.

Es en esta última dimensión donde se aprecia como propósito fundamental la formación en valores, porque al ser inherentes a las propias actividades, busca combinar el desarrollo de las actividades físicas y la satisfacción de los alumnos, lo cual lleva al logro de los objeti- vos para el fortalecimiento social y ciudadano.

Es particularmente significativo que los estudiantes le asignen prioritariamente favorabilidad al valor de la honestidad y el respeto, seguidos por la transparencia, probidad, responsabilidad, tolerancia, justicia y participación ciudadana. Señalar como prioritaria la honestidad es relevante porque sin él, carecería de sentido la clase de EF, este valor está íntimamente ligado a la búsqueda de la verdad, convirtiéndose en el motor que impulsa al individuo para alcanzar el bien de la sociedad. En cuanto al respeto, es también un valor universal que cobra especial matiz en el espacio formativo. El respeto a la persona, en primer lugar, por su misma dignidad y, por ende, el respeto a ideas, forma de ser y actuar, de las convicciones y expectativas, pero especialmente, el respeto mutuo en la interacción educativa entre compañeros y entre estudiantes y profesores. De todas maneras, es posible establecer la obtención de resultados interesantes desde el punto de vista moral y personal, especialmente cuando se dedica tiempo, esfuerzo e interés en objetivos y contenidos de este tipo. Solamente así, es viable alcanzar un sistema de valores estable hacia la socialización y el desarrollo personal de los estudiantes de secundaria, que sea extensible a sus vidas futuras fuera de la institución educativa.

Las anteriores actividades pueden contribuir a la formación de valores en las clases de EF, incluyendo otras que tengan relación con las características de los estudiantes y las posibilidades existentes en el medio donde se desenvuelvan, pero sin perder de vista la necesidad de ser concebidas intencionalmente, con objetivos y acciones encaminadas al impulso de valores, los cuales deben llegar a ser parte consciente y sistemática de la conducta de los estudiantes.

\section{Referencias}

Aguayo Rousell, H. B. (21- 25 de septiembre de 2009). Los valores en la clase de Educación Física. (X. C. InvestigaciónEducativa, Ed.) Ara 6 Educación y Valores.

Alvarado, L. Y. (2008). Características más relevantes del paradigma socio-crítico: su aplicación en investigaciones de educación ambiental y de enseñanza de las ciencias realizadas en el doctorado en educación. Universitaria de Investigación.(2), 187-202.

Antúnez, S. (2009). Prólogo de "Cómo educar en valores». Madrid: Nárcea.

Arana Ercilla, M., \& Batista Tejeda, N. (2017). La educación en valors: una propeusta pedagógica para la formación profesional. Ciencia, Tecnología, Sociedad e Innovación para el 
Desarrollo Sostenible.

Arufe, V. (2009). La educacuón en valores en el aula de educación física ¿mito o realidad? EmásF. Revista digital de educación fisica., 9, 32-42.

Aznar Quilis, N. (2015). La mejora de la conviviencia en el aula en el área de Eduación Física de la Educación Secundaria Obligatoria. Tesis Doctoral, Universidad de Valencia, Valencia (España).

Ballester Hernández, F. (27 de noviembre de 2019). Educación en valores y mejora de la convivencia: una propuesta integrada. Microsoft WordEducaciónValoresMejoraConvivencia.doc. Recuperado de http://www.diversidad.murciaeduca.es

Buxarrais, M. R., Martínez, M., Puig, J. M., \& Trilla , J. (1995). La educación moral en primaria y secundaria. Madrid: MEC-Edelvives.

Cagigal, J. M. (2006). Desarrollo de valores en la educación física y el deporte. Educación Física y Deportes. Apunts(51), 100-108.

Cañón Salinas, F. G. (2019). Percepción de la conviviencia escolar en instituciones de carácter oficial y privado de la ciudad de Ibagué. Tesis de Maestría. Universidad del Tolima, Ibagué.

Carbonell, S. J. (2000). Pedagogías del Siglo XX. Cuadernos de Pedagogía especial 25 años. Barcelona, Escpaña: CISSPRAXIS S.A.

Carbonell Ventura, T., Antoñanzas Laborda, J. L., \& López Alvarez, Á. (2018). La educación física y las relaciones sociales en educación primaria. International Journal of Devolopmental an Educational Psychology., 2(Esp. 1).

Carreras, L., \& et al. (2009). Cómo educar en valores. Materiales, textos, recursos, técnicas. . Madrid: Nárcea.

Chomsky, N. (1978). La lingúística cartesiana.. Mdrid: Gredos.

Collado Fernández, D. (2005). Transmisión y adquisición de valores a través de un programa de Educación Física basado en el juego motor, en un grupo de alumnos y alumnas de primero de educación secundaria obligatoria. . Granada, España:Tesis de doctorado, Universidad de Granada, España.

Comte, C. (2013). La contribución de las comunidades de aprendizaje al desarrollo de la competencia social y ciudadana. Tesis inédita de doctorado, Universidad de Barcelona, Barcelona.

Consejo Escolar de la Región de Murcia. (2017). El aprendizaje de los valores a través del deporte. Murcia (España): CERM.

Consejo Internacional para la Ciencia del Deporte y la Educación Física. (1999). Cumbre Mundial sobre Edeucación física. Berlín.

Contreras Jordán, O. R. (2013). Educación en valores. Universidad de Castilla. Albacete: La Mancha.

Cortina, A. (1999). Ciudadanos del mundo: Hacia una teoría de la ciudadanía. Madrid: Alianza.
Dalla Costa, G. (2014). Los valores que aporta la Educación Física, el reconocimiento y acompañamiento de las demás áreas curriculares. Artículo 428, Congreso Iberoamericano de Ciencia, Tecnología, Innovación y Educación, Buenos Aires.

Deval, J., \& Enesco, I. (1994). Moral, Desarrollo y Educación. Madrid: Grupo Anaya.

Díaz Torres, J. M., \& Rodríguez Gómez, J. M. (2008). La educación en valroes como estrategia de desarrollo y consolidación de la persona moral. Estudios sobre Educación No. 15 ESE 160, 159-169.

Escámez, J., García López, R., Pérez Pérez , C., \& Llopis, A. (2007). El aprendizaje de valores y actitudes. Teoría y práctica. Madrid: Octaedro.

Esclante Ruiz, L. A. (2014). La formación ciudadna en las clases de Educación física. EFDeportes.com. Revoista Digital., 19(198).

Estartí, A., Pascual, C., Marín, D., Martínez, C., \& Chacón, Y. (2005). Responsabilidad personal y social a través de la educación fisica y el deporte. Barcelona: Graó.

Fabelo, J. (1989). Práctica, conocimiento y valoración. La Habana: Ciencias Sociales.

Federación Internacional de Educación Física - FIEP. (2000). Manifiesto Mundial de la Educación Física. NuevaYork: FIEP.

Gairín, J., \& Roure, M. (1996). La situación de la enseñanza de la Educación Física en las enseñanzas medias. Madrid: Centro de Investigación y documentación educativa. .

García Muñoz,T. (2003). El cuestionario como instrumento de investigación/evaluación.

Gaviria Cortes, D. F., \& Castejón Oliva, F. J. (2016). Desarrollo de valores y actitudes a través de la clase de educación física. Movimiento, 22(1), 251-262.

Goetz, J., \& LeCompte, M. (1988). Etnografía y diseño cualitativo en investigación educativa. Madrid: Morata S.A.

Gómez Rijo, A. (2005). La enseñanza y el aprendizaje de los valores en la educación deportiva. Revista Internacional de Medicina y Ciencias de la Actividad Física y el Deporte, 5(18), 89-99.

Gómez, R., Rozengardt, R., \& Renzi, G. (2009). El valor formativo de la Educación Física. La Pampa, Argentina: Ponencia Congreso Internacional de Educación física Escolar.

González Lucini, F. (1990). Educación en valores y diseño curricular. Madrid: Alhambra-Longman.

Gutiérrez Sanmartín, M. (1995). Valores sociales y deportes. Madrid: Gymnos.

Gutiérrez Sanmartín, M. (2005). Manual dobre valores en educación física y el deporte. Barcelona.: Paidós.

Hernández, R., Fernández, C., \& Baptista , P. (2008). Metodología de la Investigación. México: MacGraw-Hill.

Hine, C. (2004). Etnografia virtual. Barcelona: UOC. 
Hurtado Lazo, J. R. (2010). Actitud de los estudiantes del cilco común con respecto a la asignatura de Edcuación Física. Tegucigalpa, Honduras: Universidad Pedagógica Nacional Francisco Morazán.

Igarabide Iparaguirre, I. (2014). La Educación Física como entrono de trabajo de la educación en valores en educación primaria. Teis de Grado, Universidad deValladolid, Soria.

JiménezVaquerizo, E. (2015). La educación física y los valores democráticos. EFDEportes.com Revista Digital, Año 20(210).

Jiménez, P. (2000). Modelo de intervención para educar en valores a jóvenes en riesgo a través de la actividad fisica y el deporte. Universidad Politécnica de Madrid. Madrid: UPM.

Lanni, N. (s.f.). La convivencia escolar: una tarea necesaria, posible y compleja. Monografias virtuales Ciudadanía, democracia y valores en sociedades plurales. (2 ).

LLopis, J. (1993). Los valores en la reforma del sistema educativo. Estudio para una educación en los valores. Tesis Doctoral. , Universidad de Valencia, Valencia (España).

Lopetegui Morales, R., \& Ceballo Zúñiga, J.A. (2012). Plan de actividades recretivas para contribuir a la formación ciudadana de jóvenes de 21 a 30 años de la comunidad de «El Cuncuní» en la Isla de la Juventud. EFDeportes.com, 17(172).

Ministerio de Educación Nacional. (1996). Serie lineamientos curriculares: Educación fisica, recreación y deportes. Bogotá: Men.

Ministerio de Educación Nacional. (26 de noviembre de 2019). Politica educativa para la formación escolar en la convivencia. Recuperado de www.mineducacion.gov.co

Méndez, J. M. (2001). Cómo educacr en valores. Madrid: Síntesis.

Miraflores, E. (2006). El valor como contenido de la Educación Física Escolar. Educación y Futuro, 15, 149-162.

Monzonis Martínez, N., \& Capllonch Bujosa, M. (2014). La educación física en la consecución de la comptencia social y ciudadana. Retos. Nuevas tendencias en Educación Física, Deportes y Recreación. No. 25, 180-185.

Monzonis Martínez, N. (2015). La Educación fisica como elemento de mejora de la competencia social y ciudadana. Tesis doctoral, Universidad de Barcelona, Barcelona.

Moscovici, S. (1994). Psicología Social. Barcelona, España.: Paidós.

Negret Fuentes, J. (2016). Formación ciudadana, cultura físicda y deporte: estrategia para una formación de calidad. Revista Cubana de Educación Superior, 35(1).

Organización de las Naciones Unidas para la Educación, la Ciencia y la Cultura. (2015). Organización UNESCO. Recuperado de portal.unesco.org/es/ev.phpURL_ID13150\&

Ortega, P., Gil, R., \& Minguez, R. (1996). Valores y Educa- ción. Barcelona: Ariel.

Ortega Ruiz, P., \& Minguez Vallejos, R. (2001). Los valores en la educación. Barcelona: Ariel.

Ortega, R. (s.f.). Competencias para la conviviencia y las relaciones sociales. Cuadernos de Pedagogía.(370 Monográfico), 32-35.

Parada, A. (2012). La importancia de la Educación Física. Curso 2020-2021, Educación Física - Andres Parada.

Parlebas, P. (1991). Educación Física moderna o ciencia de la acción motriz. XIII Congreso Panamerica de Educación Física. Bogotá: ACPEF.

Parra Ortiz, J. M. (2003). La Educación en valores y su práctica en el aula. Tendencias Pedagógicas 8, 69-88.

Pascual, A. (1988). Clarificación de valores y desarrollo humano. . Madrid: Narcea.

Pestaña de Martínez, P. (julio - diciembre de 2004). Aproximación conceptual al mundo de los valores. (REICE, Ed.) REICE. Revista Iberoamericana sobre Calidad, Eficacia y Cambio en Educación., 67-82.

Piéron, M., Ruiz, F., \& García, M. (2008). La opinión del alumno de educación secundaria sobre las clase de educación física. Un desafío para los profesores y los formadores. . Revista de la Facultad de Ciencias de la Educación., 159-175.

Pinzón Arciniegas, R. D. (2017). El currículum del área de la educación física como promotor de hábitos de vida saludable del colegio Pablo Tarso. Tesis de Maestría, Universidad Santo Tomás., Bogotá.

Prat, M., \& Soler, S. (2003). Actitudes, valores y normas en la Educación Física y el Deporte. Barcelona: INDE.

Puig, J. (1995). La educación moral en la enseñanza obligatoria. Barcelona: I.C.E.

Ramos, M. G. (2000 ). Programa para educar en valores. Caracas: Paulinas.

Ruiz Omeñaca, J. V. (2014). La educación en valores desde los deportes en equipo. Tesis doctoral, Universidad de la Rioja. La Rioja.

Ruiz Silva, A., \& Chaux Torres, E. (2005). La formación de competencias ciudadanas. Bogotá: ASCOFADE.

Sanchez,W.Y. (2017). La netnografía un modelo etnográfico en la era digital. Espacios, 38:1-14.

Santana, G. C., Bogoya, M. N., \& Rojas, T. G. (2010). Valores ciudadanos y democráticos: una perspectiva. REXE. Revista de Estudios y Experiencias en Educación(8).

Tallone, A. E. (2014). Educación, valores y ciudadanía. Metas Educativas 2021: la educación que queremos para la generación de los bicentenarios. Madrid: OEI- Bravo Murillo,38.

Velásquez Callado, C. (2013). Análisis de la implementación del aprendizaje cooperativo durante la escolarización obligatoria en el área de Educación Física. Tesis Doctoral, Univerisda de Valladolid, Valladolid. 